\section{G322 IV FLUIDS TO PROTECT AGAINST STEC-HUS - IMPACT OF HEALTH PROTECTION SCOTLAND GUIDELINES ON ACUTE PAEDIATRICS SERVICES}

G Bruce, V Harkins. Royal Hospital for Children Glasgow, NHS Greater Glasgow and Clyde, Glasgow, UK

\subsection{6/archdischild-2020-rcpch.278}

Introduction Acute bloody diarrhoea is associated with E.Coli enterocolitis, progressing to haemolytic uraemic syndrome (STEC-HUS) in 10-15\% of cases. Health Protection Scotland (HPS) guidelines recommend commencing pre-emptive IV fluids in children with acute bloody diarrhoea and high risk features until an E.Coli result is known to reduce progression and severity of STEC-HUS. The service impact of this change is unknown.

Aims

- Establish baseline annual presentation rate of children with acute bloody diarrhoea to RHC, Glasgow

- Evaluate management of children eligible for pre-emptive IV fluids prior to guideline implementation

- Estimate service impact of guideline implementation

Methods A microbiology search identified all stools sent from RHC (ED/CDU) between 1/3/18-1/3/19. Children with acute bloody diarrhoea and high risk features were identified by screening records of patients with bloody/diarrhoeal samples. Data regarding management/diagnosis was extracted. CMPI and IBD cases were excluded.

Results 55 patients had acute bloody diarrhoea (9\% of 577 stool samples). 8 CMPI and 7 IBD cases were excluded. Of the remaining 40, 29 had high risk features meeting criteria for pre-emptive IV fluids. 32 had blood tests and 24 had urinalysis.

Of 29 high risk patients, 22 (76\%) were admitted for an average of 2 days (excluding HUS) and 7 (24\%) were not admitted. $14(47 \%)$ received IV fluids $>24$ hours and 8 (28\%) were discharged before 24 hours. 22 (76\%) grew a bacterial pathogen (E.Coli $(n=4)$, Campylobacter $(n=15)$, Salmonella $(\mathrm{n}=3))$ and $3(10 \%)$ developed STEC-HUS. Mean time to stool culture result was 47.8 hours.

Of 11 without high risk features, 1 grew a bacterial pathogen (Campylobacter).

Conclusion In this cohort, implementing HPS guidelines would necessitate 22 additional inpatient hospital days for IV fluids, 8 additional blood tests and 16 additional urinalysis tests. High risk features appear sensitive for pathogenic bloody diarrhoea and captured all STEC-HUS/E.Coli cases.

\section{G323 DECISION-MAKING IN NEONATAL INTESTINAL FAILURE: PALLIATIVE OR ACTIVE CARE?}

1,2PA Cairns. 'Centre for Ethics in Medicine, University of Bristol, Bristol, UK; ${ }^{2}$ The Neonatal Intensive Care Unit, University Hospitals Bristol NHS FT, Bristol, UK

10.1136/archdischild-2020-rcpch.279

Survival rates for neonatal intestinal failure (IF) have improved significantly over the past decade, however there is no consensus for decision-making among UK paediatric subspecialists.

Aim The aim of this study was to describe health professionals' decision-making regarding palliative versus active management in neonatal IF and to examine variation between subspecialties.

Methods An electronic survey was designed to explore several neonatal scenarios. It was sent to neonatologists, paediatric surgeons and gastroenterologists on two occasions. In addition to being asked whether they would recommend active or palliative care or allow the parents to decide; they were asked whether they would consider the treatment morally obligatory or impermissible, that is authority should be sought from the courts if there is parental disagreement with the professional opinion.

Results The survey was completed by 147 respondents of which 75 (21\% were neonatologists, 26 (18\%) paediatric surgeons and 26 (18\% surgeons) with 20 (13\%) other.

In a term baby with total gut Hirschsprung's, $81 \%$ of gastroenterologists would recommend active care (regardless of parental decision in 34.6\%) compared to $46 \%$ of surgeons and $35 \%$ of neonatologists. No gastroenterologist would recommend palliative care while $23 \%$ of both neonatologists and surgeons would.

Similarly, in a 28 -week gestion neonate with bilateral brain haemorrhages and IF secondary to necrotising enterocolitis (NEC) $77 \%$ of surgeons and $73 \%$ of neonatologists would recommend palliative care compared with only $27 \%$ of gastroenterologists.

For a term baby with IF secondary to volvulus, 58\% of gastroenterologists estimated that she would have a fiveyear survival of $>80 \%$ compared to $11.5 \%$ of surgeons and only $2.7 \%$ of neonatologists. Similarly, for a 26 week gestation baby with IF secondary to NEC only $11.5 \%$ of surgeons and $2.6 \%$ of neonatologists believed her 5-year survival would be $>60 \%$ compared to $59 \%$ of gastroenterologists.

Conclusion Paediatric subspecialists have very different views about long-term outcomes and the appropriateness of active or palliative management in neonatal IF, with gastroenterologists being consistent more optimistic than surgeons or neonatologists. This is likely to lead to unjustified variation in counselling and choices given to parents.

\section{G324 EXPRESSION OF NEURONES AND NEURONAL PRECURSORS IN THE TRANSITION ZONE OF SHORT SEGMENT HIRSCHSPRUNG'S DISEASE}

${ }^{1} \mathrm{~J}$ Doherty, ${ }^{1} \mathrm{~S}$ Chhabra, ${ }^{1} \mathrm{M}$ Smith, ${ }^{1} \mathrm{D}$ Edgar, ${ }^{2} \mathrm{~S}$ Almond, ${ }^{1,2}$ SE Kenny, ${ }^{1} \mathrm{~B}$ Wilm. ${ }^{1}$ Institute of Translational Medicine, University of Liverpool, Liverpool, UK; ${ }^{2}$ Alder Hey Children's Hospital

\subsection{6/archdischild-2020-rcpch.280}

Aims Hirschprung's Disease (HSCR) is a congenital disorder of dysfunctional enteric nervous system (ENS) development, resulting in distal aganglionosis of the bowel. The cellular composition of the transition zone (TZ), the region between the distal aganglionic bowel and normally innervated ganglionic bowel, is not well understood. Some children have resectional surgery in which TZ bowel is left in situ with variable effects on functional outcome. Understanding the cellular anatomy of this region may aid in understanding the aetiology of HSCR and the effects of TZ pull-through on outcome, in addition to permitting the development of safe and effective use of ENS progenitor cells as therapy. This work aimed to characterise the TZ of short segment HSCR bowel, by assessing the expression of ENS and 
neuronal markers by immunofluorescence and quantitative PCR(qPCR).

Methods Human bowel was collected following a pull-through procedure in four patients with short segment HSCR. Protein expression of ENS and neuronal markers (p75, SOX10, PHOX2b, Hu and GLUT1) in ganglionic, TZ and aganglionic region of the bowel were visualised through immunofluorescence and mRNA levels of the corresponding markers were quantified using qPCR.

Results Immunofluorescence analysis showed a gradual loss of SOX10, PHOX2b and $\mathrm{Hu}$ protein in the lower TZ and absence in the aganglionic region. However, generally the marker expression presented with inter-patient variability within the TZ. By contrast, GLUT1 was highly expressed in the perineurium of thickened nerve trunks, characteristic of the aganglionic region. Perineurial structures positive for GLUT1 were also visualised in the TZ, but to a lesser extent than the aganglionic region. This observation corresponded with a decrease in mRNA levels of p75, SOX10, PHOX2b and $\mathrm{Hu}$ from the ganglionic to the aganglionic region and an increase in GLUT1.

Conclusion This work displayed a trend of decreasing number of cells expressing ENS and neuronal markers from the ganglionic to aganglionic region, through the TZ. However, our analysis reveals inter-patient variability in the cellular composition of HSCR bowel, especially in the TZ. Possibly explaining the variable functional outcome for HSCR children with TZ pull-through.

\section{G325(P) A SINGLE-CENTRE AUDIT EVALUATING THE APPROPRIATE USE OF DMSAS FOR THE INVESTIGATION OF PAEDIATRIC UTI}

S Ramachandran, I Hasan, P Set. Radiology Department, Cambridge University Hospitals NHS FT, Cambridge, UK

\subsection{6/archdischild-2020-rcpch.281}

Aims Technetium-99m-labeled dimercaptosuccinic acid (DMSA) scintigraphy represents an important imaging modality in investigating select paediatric patients with urinary tract infection (UTI). Recently updated NICE guidelines outline the indications for DMSA according to age group. This audit aimed to assess adherence to these guidelines and evaluate their reliability.

Methods Using PACS, we retrospectively evaluated all firsttime DMSAs performed at a tertiary centre to investigate paediatric UTI over a period of 24 months. Data collected included patient age, clinical justification, timing of the scan and abnormalities reported.

Results 105 patients underwent DMSAs (mean age: 4.0 years, range: 4 months-15.6 years). Of these, 85 cases $(81.0 \%)$ adhered to NICE guidelines. Of the 20 non-adherent cases, 8 $(40 \%)$ misinterpreted the definition of recurrent UTIs, 8 (40\%) were performed inappropriately after a solitary UTI, and $4(20 \%)$ misinterpreted the definition of an atypical UTI. Adherence was poorest for patients under 6 months at $71.4 \%$. NICE guidelines had a $88.9 \%$ sensitivity $(95 \%$ CI $65.3-$ 98.6\%); 20.7\% specificity (95\% CI $12.8 \%-30.7 \%), \quad 18.8 \%$ PPV (95\% CI 16.0\%-22.0\%); and 90\% NPV (95\% CI 69.697.3\%).

Conclusion We demonstrate relatively consistent adherence to NICE guidelines with some room for improvement. We advocate improving awareness amongst referring clinicians on the guidelines, particularly for the youngest age group and on what constitutes a recurrent or atypical UTI. Improving adherence ensures that unnecessary DMSAs are avoided, precluding radiation exposure and emotional stress. Although limited by our small sample size, we show good sensitivity but poor specificity of NICE guidelines.

\section{G326(P) INVESTIGATION AND MANAGEMENT OF PAEDIATRIC UROLITHIASIS - A UK SINGLE CENTRE EXPERIENCE}

${ }^{1}$ A Duncanson, ${ }^{2} S$ Hotonu. 'Paediatric Nephrology, Great North Childrens' Hospital, Newcastle Upon Tyne, UK; ${ }^{2}$ Paediatric Urology, Great North Childrens' Hospital, Newcastle Upon Tyne, UK

\subsection{6/archdischild-2020-rcpch.282}

Aim To evaluate our centre's experience on the management of urinary tract stones in children according to current European Association of Urology (EAU) guidelines.

Methods A retrospective review of all cases of urolithiasis treated in our tertiary paediatric centre. This encompasses a 6 year period (June 2013- May 2019). Data collected included patient demographics, urinary and biochemical workup, stone burden and analysis, surgical management, outcomes of treatment and follow up. Compliance with EAU guidelines was also audited.

Results Thirty seven patients were identified (22 males). Median age was 8 years (range 1-16 years). Mean weight was $31.5 \pm 8$ kilograms, with 3 patients being above the 75 th centile. Risk factors for stone formation were identified in twenty four patients: four patients (10.8\%) were on medication known to precipitate urolithiasis, two patients $(5.4 \%)$ had a family history of renal stones, five patients $(13.5 \%)$ had a metabolic cause, ten patients $(27 \%)$ had a history of recurrent urinary tract infections and three patients $(8.1 \%)$ had a combination of risk factors. The remaining thirteen patients were idiopathic stone formers. Nine patients had bilateral renal stones, twenty five unilateral, and three had bladder stones. Majority of stones were calcium based. Twenty two patients $(59.4 \%)$ had surgical management of stones, with 3 patients requiring repeat procedures for high stone burden. Eight patients $(21.6 \%)$ had impairment of renal function secondary to stones.

Conclusions Management of paediatric urolithiasis is complex and is associated with significant morbidity. The majority of patients have clear risk factors for stone formation. Investigation according to European guidelines provides the means to identify such patients. Further multicentre collaborative studies are warranted.

\section{G327(P) AN AUDIT OF ACUTE KIDNEY INJURY IN CHILDREN}

${ }^{1} \mathrm{R}$ Collier, ${ }^{2} \mathrm{R}$ Holt, ${ }^{2} \mathrm{CA}$ Jones, ${ }^{3} \mathrm{~S} J \mathrm{McW}$ illiam. ${ }^{1}$ Medical School, University of Liverpool, Liverpool, UK; ${ }^{2}$ Department of Nephrology, Alder Hey Children's NHS FT, Liverpool, UK; ${ }^{3}$ Department of Women's and Children's Health, University of Liverpool, Liverpool, UK

\subsection{6/archdischild-2020-rcpch.283}

Aims Acute Kidney Injury (AKI) in hospitalised children is significantly associated with increased morbidity and mortality. The British Association of Paediatric Nephrology AKI management guidelines recommend early identification of patients at 